\title{
UNA MIRADA A LA DIDÁCTICA DESDE LA FORMACIÓN CATEGORIAL. PERSPECTIVA DE ANÁLISIS DIDÁCTICO PARA LA EDUCACIÓN FÍSICA ${ }^{1}$
}

\author{
Luz Elena Gallo Cadavid \\ Universidad de Antioquia. Colombia
}

\begin{abstract}
Calias, le dije, si tus dos hijos fueran potros o becerros, tendríamos que tomar un cuidador de ellos y pagarle; éste debería hacerlos aptos y buenos en la condición natural que les es propia, y sería un conocedor de los caballos o un agricultor. Pero, puesto que son hombres, ¿qué cuidador tienes la intención de tomar?, ¿quién es conocedor de la perfección humana?
\end{abstract}

Platón, Apología de Sócrates (1997)

\section{INTRODUCCIÓN}

Con base en la anterior apología de Sócrates, ¿quién puede 'ayudar' a la perfección humana?: la pedagogía o ciencia de la educación, entendida ésta como un campo donde se reflexiona sobre la educación y la formación. Por ello, este texto se desarrolla desde la siguiente pregunta conductora: ¿Cómo pensar el enseñar y el aprender que gobierna la didáctica a partir de la formación categorial? Esta pregunta muestra el camino que se recorrerá en el bosque de la didáctica, pues como dice Heidegger (2003: 10) :“En el bosque hay caminos, por lo general medio ocultos por la maleza, que cesan bruscamente en lo no hollado". De los caminos que llevan a la didáctica, se toma la formación categorial, que algunos leñadores y guardabosques ya conocen; se trata de transitar en él fundamentalmente a través de los contenidos, con el fin de des-cubrir en el viaje cuándo un contenido (Inhat) tiene contenido formativo (Gehalt). Viaje, que también es un pretexto para que la Educación Física devele lo formativo en sus contenidos por medio de la teoría de la formación categorial.

Cabe resaltar, entonces, que este texto transita por tres pasajes. En el primero, se inicia el camino, a manera de introducción, con la tesis de que el ser humano es un ser necesitado de educación, la cual se orienta desde una perspectiva antropológico-pedagógica. En el segundo, se presenta de manera interpretativa la teoría de la formación categorial a partir de los autores Josef Derbolav y Wolfgang Klafki, quienes han desarrollado dicha teoría en forma sistemática. En el tercero, se infiere cuándo un contenido tiene contenido formativo, de tal manera que el camino toma un trazado diferente porque deja de ser segmentado y fragmentado y empieza a encontrar trochas que podrían ser útiles para un análisis didáctico en la Educación Física.

\footnotetext{
1 Este texto se enmarca en la tesis doctoral "Elementos para una fundamentación antropológico-pedagógico y fenomenológica del movimiento humano y la corporalidad articulados con la formación", que actualmente realizo en el Doctorado en Educación, con la dirección del Dr. Phil. Andrés Klaus Runge Peña.
} 


\title{
1. EL INICIO DEL CAMINO: EL SER HUMANO ES UN SER CON NECESIDAD DE EDUCACIÓN
}

La antropología pedagógica estudia al hombre/mujer como ser formable, capacitado y necesitado de educación. La necesidad de educación hace que el ser humano tenga perfectibilidad o capacidad de perfeccionarse, subjetivarse, autorrealizarse, hacerse cargo de sí, descubrir posibilidades y desarrollar potencialidades.

Según Speck y Wehle (1981: 528),

"El hombre aparece al comienzo de su vida como un ser dependiente y no desarrollado. El niño recién nacido es el más desprotegido de la naturaleza (Herder); es 'un ser orgánico deficiente', insuficiencia que no se puede remediar sin ayuda ajena (Gehlen); la comparación del estado de desarrollo del hombre y del animal en el momento del nacimiento da pie a Portmann para caracterizar al lactante humano como parto fisiológico prematuro, que sin el seno materno social no está capacitado para vivir".

Desde la antropología-pedagógica, ser humano es tener que hacerse constantemente a través de la educación, pues, mediante la paideia, se realiza el inacabable proyecto humano. El hecho de que el ser humano es un 'ser carente' que no está 'acabado', que es un 'ser desvalido, necesitado y expuesto' es, a su vez, un ser carente y conciente de esa carencia, es un ser necesitado de educación que tiene la fuerza que lo empuja a un continuo incesante de hacer y hacerse. En la didáctica crítica (Klafki, Derbolav, Martínez, entre otros) se dice que el hombre es un-ser-haciéndose, en devenir, un ser ubicado entre lo finito y lo infinito, histórico, aprendiendo siempre y constituyéndose en ese aprendizaje como inacabado.

La educación tiene que ver con el proceso de estructuración de la personalidad del individuo, en tanto es un ser flexible, maleable, cambiable y con capacidad de auto-transformación. Es precisamente a partir de la relación con el otro, de la 'ayuda' del otro, como se actualizan los modos de ver y de obrar, se desarrolla la capacidad de expresión, de la individualidad, se despierta la capacidad de tener vivencias significativas, se potencia una acción responsable consigo mismo, con el otro y el mundo, se integra a una cultura y, a su vez, se transforma.

\section{2. ¿QUÉ HAY SIGNIFICATIVO EN EL CAMINO?: UN RECLAMO POR LO FORMATIVO EN LOS CONTENIDOS}

\begin{abstract}
Enseñar es aún más difícil que aprender. Se sabe esto muy bien, mas, pocas veces se lo tiene en cuenta.

¿Por qué es más difícil enseñar que aprender? No porque el maestro debe poseer un mayor caudal de conocimientos y tenerlos siempre a disposición. El enseñar es más difícil que aprender porque enseñar significa: dejar aprender. Más aún: el verdadero maestro no deja aprender nada más que el aprender. Por eso también su obrar produce a menudo la impresión de que propiamente no se aprende nada de él, si por 'aprender' se entiende nada más que la obtención de conocimientos útiles.
\end{abstract}

Heidegger (1978: 20)

Según Speck y Wehle (1981: 270) "la teoría de la formación tiene el quehacer de poner en claro los presupuestos antropológicos del hecho de la formación, fijar el camino que tiene que recorrer el hombre en el proceso de hacerse 'hombre', e indicar de qué manera se le puede ayudar en este empeño mediante un influjo metódico, con arreglo a un plan". De igual forma, toda teoría de la formación debe partir de la relación 
del hombre con el mundo. De esa relación primigenia, que es constitutiva de la formación en general, hace parte la teoría de la formación categorial ${ }^{2}$.

La teoría de la formación categorial proviene de la reflexión didáctica (Speck y Wehle (1981: 272) y se fundamenta en la teoría de la formación y la didáctica de Copei, Flitner, Weniger y Wagenschein y fue desarrollada en forma sistemática por Josef Derbolav y Wolfgang Klafki a finales de los años 50 y comienzos de los años 60 del siglo XX. Klafki parte del concepto de formación de Humboldt ${ }^{3}$ y uno de los grandes aportes es el haberle dado gran importancia al concepto de formación dentro de la didáctica. La tarea del maestro, preceptor $(\delta ı \delta \alpha \sigma \kappa \alpha \lambda \circ \zeta)$ consiste en trabajar sobre los contenidos y explotar su aspecto formativo. Por su parte "Derbolav, recurriendo al pensamiento de Hegel, parte de que la formación categoría central del proceso educativo y de su teoría- ha de entenderse como comportamiento del educando con el mundo y consigo mismo" (Speck y Wehle, 1981: 632). Para este autor el concepto de formación está relacionado con mediación, donde mismidad y mundo no se reducen uno al otro, sino que se han de captar en un constante 'movimiento de mediación', que es un 'llegar a sí mismo en el otro'.

En este contexto, la mediación se convierte en un eje central para la formación categorial, pues el maestro es el mediador, permite que el otro se apropie de sí mismo, se transforme y se convierta en sujeto. En esta teoría de la formación, el maestro se debe apropiar de un contenido, de tal manera que le reconozca el sentido que tiene y lo oriente con el fin de que, a partir de esa mediación, cobre un significado formativo. Es pues una relación dialéctica, en la que se trata de mediar entre las exigencias del mundo objetivo que se expresa en el saber producido por la humanidad y que mediante la enseñanza y el aprendizaje el sujeto llegue a ser él mismo, se autorrealice, autodetermine, se-haga-sujeto y construya subjetividad.

Formación para Derbolav (citado por Speck y Wehle, 1981: 273) es "la mediación del yo a través de lo otro; en ello el yo viene dado al educador como el respectivo tú e individuo concreto, y lo otro como las respectivas y concretas objetivaciones y esferas de saber y de cultura". La acción mediadora implicada en el sentido de la formación se designa como 'categorial'. Son categorías de formación "las estructuras de normas o contenidos deónticos específicos del sector, que se presuponen en los contenidos de objetivos de las ciencias y que el yo elabora para sí en el diálogo de la formación, y en los que al mismo tiempo él se encuentra individualmente en forma de un determinado horizonte de responsabilidad" (Speck y Wehle, 1981: 273).

La formación está relacionada tanto con los contenidos de las ciencias o de la disciplina, como aquellos contenidos en el que el yo se estructura, en los que toma conciencia-de-sí y del mundo. En este sentido, formación es el proceso de autorrealización del individuo en la compenetración consigo mismo y con el mundo.

\footnotetext{
${ }^{2}$ Speck y Wehle (1981: 267) distinguen cuatro teorías de la formación que tienen relevancia para el pensar pedagógico de la actualidad: la formación formal, la formación categorial, la teoría dialogística de la formación y la formación técnica.

${ }^{3}$ Para Humboldt, la formación se dirige a la mediación entre condiciones histórico-sociales externas y condiciones individuales internas. Para el logro de esos procesos se necesitan una libertad individual y unas posibilidades de formación alcanzadas de un modo social y múltiple. Solo así pueden ser elaboradas las pretensiones ligadas a la meta de una formación lo "más elevada y proporcional posible" y los conflictos. Los resultados de esos procesos de formación se encuentran abiertos al futuro. La apertura al futuro significa: lo desconocido y lo inseguro del futuro y lo inacabado de la formación humana son tenidos por elementos constitutivos en el proceso de formación, en el que las inseguridades que se dan en él no se alcanzan a cubrir por medio de supuestas seguridades (Wulf, 2004: 54).
} 
De otra parte, según Klafki, autor representativo de la didáctica crítica, la teoría de la formación categorial está orientada en el sentido didáctico. Esta teoría establece un enlace entre formación categorial y pensar didáctico y, a partir de la comprensión de esta conexión, esboza una 'nueva didáctica', pues supera la escisión entre objetivo y subjetivo, al establecer una fusión de sujeto y objeto. La formación es para el autor apertura de una realidad material y espiritual a una persona (aspecto objetivo), pero esto significa también apertura de esta persona a esa su realidad (aspecto subjetivo). La educación como apertura exige una tarea, 'un ir hacia' entre lo que se es y lo que podría-llegar-a-ser, en este sentido implica hacerse-hombre, hacerse-mujer; y por ello "la formación (Bildung) lleva consigo conceptos como autodeterminación, libertad, emancipación, autonomía, mayoría de edad, razón, autoactividad" (Klafki, 1987).

Este autor toma como referencia a Wilhelm von Humboldt, desde donde entiende que en la formación entran en intercambio la objetividad del mundo y la subjetividad del individuo. Humboldt, en su teoría de la formación establece tres principios: fuerza, yo/mundo y unidad, principios que son esencialmente antropológicos. El principio de fuerza dice: lo que el hombre es en su esencia, lo es como fuerza. El hombre es activo, dispone de espontaneidad y fuerzas que se pueden manifestar; porque el hombre se concibe como fuerza y porque tiene en el mundo un enfrente, en el que puede desarrollar y aumentar sus fuerzas individuales, es posible la formación. El principio yo/mundo reúne el pensamiento del autor en cuanto a la formación, puesto que el hombre para poder formarse, realizarse a sí mismo, tiene que ponerse en relación con el mundo que está frente a él; y para la realización de sí mismo, el yo tiene que formar una unidad completa. El principio de la unidad se refiere a que la formación depende de formar todas las fuerzas proporcionalmente, porque sólo así puede garantizarse una participación del mundo lo más completa posible y la formación de menos fuerzas, sólo puede construir una relación parcial con el mundo 4 .

Según estos principios, se puede decir que si la Educación Física sólo privilegia el desarrollo de la fuerza física, "en consecuencia la formación del hombre es parcial e inarmónica, para enfrentar esta amenaza, la Educación Física debe formar las fuerzas corporales" (Meinberg, 1993). La Educación Física establece diferencia entre la fuerza 'física' y las 'fuerzas corporales', el privilegio de la fuerza física no concibe al ser humano como una totalidad, ya que lo descompone en partes para trabajar con un cuerpo de manera segmentada, al punto que se toma el cuerpo del otro como máquina para manejarlo y modificarle su funcionamiento. Este moldeamiento del cuerpo-objeto (Körper) parte de concebir el cuerpo-máquina compuesto por diferentes sistemas (óseo, muscular, cardiovascular, respiratorio, endocrino, nervioso) en los cuales se pretende hacer adaptaciones y modificaciones a través del movimiento.

Además, propio de la racionalidad, el cuerpo se toma como objeto al descuartizarlo en partes para ser intervenido a través del movimiento, pues se trabaja separadamente, por ejemplo, por grupos musculares y ello obedece a planes de entrenamiento que potencian su rendimiento. Desde esta concepción, la formación de la unidad no es posible si se concibe al cuerpo-objeto, ya que hay que considerar al ser humano como un todo indivisible. Para ello hay que trascender la metáfora mecánica del

\footnotetext{
${ }^{4}$ Desde la Educación Física, Ommo Grupe (1976) se acoge al principio de la unidad de Humboldt y plantea que se trata de pasar en la Educación Física de un pensamiento dualista, del cuerpo-cosa (Körper) a una formación centrada en la unidad, en la corporalidad (Leiblinchkeit) del hombre.
} 
cuerpo y del movimiento, donde se le ha dado más valor al cuerpo como algo objetivo, mecánico, funcional e instrumental, el cual es manejable, adaptable y modificable, por un cuerpo-sujeto (Leib), como unidad sintiente y fungiente. El cuerpo fungiente (ser-cuerpo) es el cuerpo que siente, vivencia y experimenta, "se trata de un cuerpo que intuye, experimenta, padece, expresa y siente" (Runge, 2002).

Con base en estos principios esbozados en Humboldt de fuerza, yo/mundo y unidad, para Klafki las teorías de la formación son poco claras frente a los contenidos porque no explicitan cuáles podrían ser los contenidos 'formativos' que ayudan desde la relación yo/mundo a la formación de las fuerzas y potencias. Este autor hace, además, una crítica contra aquellos contenidos enciclopédicos (objetivos) que se imparten sin tener en cuenta el contexto, el momento histórico y la problemática social, y que no tienen un trasfondo sobre el significado (subjetivo) que tienen los contenidos para la formación del individuo. De esta manera, para Klafki (citado por Runge, 2004), la didáctica es "el resultado de un proceso hermenéutico que presupone la comprensión y la capacidad de transmitir. El maestro se debe apropiar entonces de un contenido de tal manera que reconozca en él lo significativo y pueda trasmitirlo con el fin de que, a partir de esa mediación, tenga lugar el proceso formativo".

Hay en la teoría de la formación categorial una base hermenéutica, al poner como eje central el sentido y la compresión, pues la formación categorial propone que los contenidos se conviertan en formativos, en la medida en que sean pensados por la reflexión didáctica como significativos para la formación del sujeto. Es decir, se trata de revelar lo formativo que se encuentra en los contenidos; para ello, el maestro debe reconocer el contexto de sentido que lo abarca para orientar los contenidos desde una perspectiva formativa; y debe reconocer las problemáticas del contexto y relacionarlas con la enseñanza y con el aprendizaje.

\title{
3. ¿CÓMO DES-CUBRIR CUÁNDO UN CONTENIDO TIENE CONTENIDO FORMATIVO?
}

\begin{abstract}
No existe preocupación por uno mismo sin la presencia de un maestro, pero lo que define la posición del maestro es que aquello de lo que él se ocupa es precisamente el cuidado que pueda tener sobre sí mismo aquel a quien él sirve de guía.

El maestro es quien se cuida del cuidado del sujeto respecto a sí mismo y quien encuentra en el amor que tiene por su discípulo la posibilidad de ocuparse del cuidado que el discípulo tiene de sí mismo. Al amar de forma desinteresada al joven discípulo, el maestro es el principio y el modelo del cuidado de uno mismo que el joven debe tener de sí en tanto que sujeto.
\end{abstract}

Michel Foucault (2002)

Para intentar des-cubrir cuándo un contenido tiene contenido formativo, es preciso hacer un giro (Kehre) y ver-a-través de esta perspectiva conceptual una nueva forma de concebir los contenidos; es pasar de aquellos que ponen el acento en una racionalidad objetivante, que como forma de pensar aleja al individuo de sí mismo en la medida en que el interés se concentra exclusivamente en la enseñanza de los saberes, hacia una nueva orientación formativa, que toque al individuo en el Ser, al sí mismo. 
Siguiendo el hilo conductor de la teoría de la formación categorial, es preciso señalar las inferencias que tendría la ruta de análisis didáctica ${ }^{5}$ para comprender cuándo un contenido tiene contenido formativo:

El problema al que se ha de enfrentar desde esta teoría de la formación es cómo integrar todos los esfuerzos educacionales para 'ayudar' a que el otro se haga a sí mismo, de acuerdo con las situaciones del contexto socio-histórico particular. Esto señala un 'ideal' de formación donde "la educación debe entenderse como liberación del individuo en formación, no como sumisión del mismo; la educación se revela además como una necesidad, pues sin asistencia educacional no se logra la actualización de la personalidad" (Speck y Wehle, 1981: 721).

Siguiendo a estos autores, la categoría antropológica de la personalidad, en este escrito, tiene como finalidad enfocar el obrar pedagógico como un proceso que facilite las condiciones desde las cuales el individuo en formación pueda actualizar su ser personal, es entonces una acción mediadora la que se debe generar en el encuentro educativo.

Con respecto a los objetivos, algunos autores de esta línea de pensamiento le confieren un valor crítico a la educación, como la idea de la ilustración de Kant, en 'la salida del hombre de la minoría de edad' y cuya consecución corresponde, según él, a la educación; y el derecho de todo hombre al desarrollo de todas sus posibilidades y la autodeterminación (Pestalozzi, Humboldt).

Con base en estos presupuestos pedagógicos, Klafki (citado por Rodríguez, 1997: 135) elabora una conceptualización de la didáctica crítica, señalando como objeto:

"Por una parte, las manifestaciones y razones de los obstáculos que se oponen a la enseñanza y al aprendizaje en cuanto desarrollo de la capacidad de autodeterminación, codeterminación y solidaridad y, por otra, las posibilidades de realizar, determinar, proyectar y experimentar tales procesos de enseñanza y aprendizaje".

Los objetivos, en esta teoría de la formación, deben estar dotados de sentido en tanto sean significativos para la formación del individuo, que 'ayuden' a la autorrealización, autodeterminación, a la comprensión de sí mismo y a tener conciencia social.

Con respecto a los contenidos y de acuerdo con la formación categorial, para Klafki los contenidos doctrinales son los que cobran forma en el 'sentido formativo'.

"Contenidos doctrinales sólo son aquellos a los que es inmanente un saber formativo. Este representa un modo de saber en el que no sólo se aclara y reconoce algo objetivamente, sino que constantemente se instituye una reflexión personal sobre lo explicado; un modo de saber en el que no sólo se incorpora a la memoria un complejo intencional, sino que al mismo tiempo se recoge una ganancia estructural, se adquiere una capacidad, se ilumina una nueva posibilidad, se experimenta un impulso práctico; un modo de saber, por consiguiente, que con el progresivo conocimiento del objeto lleva también adelante el conocimiento y dominio de uno mismo; en una palabra: un modo de conocimiento capaz de aportar una contribución a la mayoría de edad y emancipación del que aprende" (Derbolav. Citado por Speck y Wehle, 1981: 273)

\footnotetext{
${ }^{5}$ Para Klafki (1990: 87), la didáctica en sentido amplio, abarca como investigación y teorización cuatro dimensiones fundamentales: objetivos (intenciones o propósitos de enseñanza), los contenidos, las formas de organización y realización (donde la relación entre procesos de enseñanza y aprendizaje debe ser entendida como una relación de interacción). Esta es la perspectiva de la metódica, y los medios de enseñanza.
} 
Con la formación categorial se trata de que el estudiante adquiera categorías, o conceptos fundamentales que sirven como presupuestos y principios de conocimiento y de comprensión del mundo, de manera que le sean útiles y se puedan aplicar. Una visión reduccionista de los contenidos la constituye el considerar que éstos se refieren únicamente a los saberes específicos carentes de contenido formativo (Gehalt).

En esta teoría de la formación, Klafki identifica tres principios didácticos de lo elemental, lo fundamental y lo ejemplar, que permiten delimitar los contenidos y argumentar por qué determinados contenidos deben o no ser enseñados.

El principio de lo elemental se hace visible y reconocible en la medida en que es aplicable a muchas otras cosas. El principio de lo fundamental que se refiere a la mediación y transmisión de experiencias fundamentales y de apreciaciones al educando que le permiten, mediante la experiencia, reconocer las cosas. Finalmente, tenemos la dimensión de ejemplar. Se trata de que lo elemental y lo fundamental se tiene que conseguir mediante un muy buen ejemplo que deje una impresión profunda. Principio ejemplar son aquellos contenidos que no sólo existen para sí, sino que se abren o permiten abrirse a otros contenidos. Los contenidos ejemplares remiten más allá de sí, le dan de este modo una estructura al campo de aprendizaje y contribuyen a que haya orden en la cabeza de los educandos (Klafki, citado por Runge, 2004).

A partir de estos principios que plantea el autor para orientar los contenidos, nos ofrece algunas dimensiones que se pueden tomar como unidades de análisis desde un punto de vista teórico-formativo para la categoría didáctica de los contenidos, como son: el significado ejemplar del contenido, importancia para el presente, importancia para el futuro, estructura del contenido y asequibilidad del contenido ${ }^{6}$. De acuerdo con este trayecto de análisis de los contenidos, se encuentra una relación directa con los componentes de problema y objetivos antes descritos, pues no se trata de la enseñanza de contenidos enciclopédicos, de los cuales el estudiante se ha de apropiar, sino de situar los contenidos en un contexto, los cuales deben estar relacionados con el individuo, sus fuerzas (en términos de Humboldt), sus

\footnotetext{
${ }^{6}$ Estas unidades de análisis son denominadas por Runge (2004) como dimensiones y tienen unas preguntas que facilitan su comprensión. Este dispositivo puede ser útil para hacer un análisis didáctico en la forma como se seleccionan los contenidos para la formación de los Licenciados en Educación Física, para los contenidos que se sugieren en las guías curriculares y para los Lineamientos Curriculares de la Educación Física:

El significado ejemplar del contenido: ¿Qué contexto general de sentido o del mundo soporta o abarca ese contenido? (esta dimensión va en contra de un plan de contenidos enciclopédico) ¿Qué fenómeno fundamental, principio básico, criterio, problema, método, técnica, actitud se puede comprender de una manera ejemplar a partir de la relación con ese contenido? ¿De qué debería ser ejemplar, representativo, típico ese tema? ¿En dónde se puede hacer fructífero posteriormente el aporte de ese tema en tanto totalidad o en elementos particulares (apreciaciones, representaciones, conceptos valorativos, métodos de trabajo, técnicas)?

Importancia para el presente: ¿Qué importancia tiene el respectivo contenido en la vida espiritual del niño o del educando? ¿Qué importancia debería tener tal contenido desde el punto de vista pedagógico?

Importancia para el futuro: ¿En qué consiste la importancia del tema para el futuro del niño o del educando?

Estructura del contenido: ¿Cuál es la estructura del contenido -según las preguntas pedagógicas de la primera, segunda y tercera dimensiones-? ¿Cuáles son los momentos particulares del contenido y en qué conexión se encuentran esos momentos particulares? ¿En una conexión lógica, fáctica? ¿Tiene niveles el contenido? ¿Tiene diferentes niveles de sentido y de importancia? ¿Pueden ser comprendidos esos niveles de una manera independiente entre sí? ¿En qué contexto mayor se encuentra ese contenido? ¿Qué propiedades del contenido podrían hacerle difícil al educando su apropiación? ¿Qué se tiene que mantener como saber mínimo para que el contenido pueda ser tenido como un contenido espiritual "vivo" y efectivo? (Esto se dirige en contra de la reducción didáctica en la que la estructura de la disciplina especial permanece contenida, pero sólo reducida de conformidad con la edad).

Asequibilidad del contenido: ¿Cuáles son los sucesos, situaciones, intentos a partir de los cuales los niños de esa clase, de ese nivel pueden, de una manera interesada, cuestionar, comprender, contrariar, intuir, acceder a la estructura del respectivo contenido? ¿Cuáles son las situaciones, observaciones intuiciones, intentos, modelos apropiados para que los educandos den respuesta de una manera autónoma sobre la estructura de ese contenido?
} 
potenciales, sus posibilidades, su devenir histórico, sus condiciones sociales, su experiencia de vida, sus procesos de adaptación y sus modos de aprendizaje. De tal manera que, a través de los contenidos, la realidad se abre al estudiante y, a su vez, el estudiante se abre a la realidad, puesto que el estudiante no se enfrenta sólo a un contenido cultural y concreto, sino que deberá reconocer cómo lo forma el contenido como sujeto.

Cuando Roth (1970: 28) se pregunta por lo formativo en el objeto, hace alusión a la misma preocupación que acompaña este escrito. Al respecto dice que:

"Lo formativo es aquello que conduce a un experimentar valores, aquello que crea necesidades espirituales, que espiritualiza las fuerzas vitales, que forma los sentimientos, que despierta la cultura. El contenido formativo está en la fuerza, despertadora de interés y formadora de los sentimientos que el objeto posee. Aquellos aspectos del objeto en los cuales se pueden desarrollar los intereses espirituales y los sentimientos, pasan a ser el centro del conocimiento. Es una consideración sobre la 'humanidad' del objeto, sobre su fuerza para transformar las almas, su sabiduría, su fuerza confortadora, su fuerza trágica, su grandeza, su exaltación, sublimación, etc. Los momentos formativos en el objeto son aquellos que atraen el interés vital hacia ellos, que apresan el sentimiento y el ánimo, pero que, en la ocupación con el objeto, y esto es lo importante, transforman, es decir, dirigen hacia valores más altos y atan a éstos, o sea, moralizan y espiritualizan".

Se encuentra también en Roth una intención de fusionar objeto/sujeto, pues la consideración que hace sobre el contenido formativo no es menos importante que la comprensión de su contenido puramente objetivo. Aunque el contenido de un bien cultural se le abre sólo a aquel que una vez fue tocado por él, en lo mas íntimo, es decir, quien asocia un contenido cultural como algo realmente significativo para sí.

El contenido se convierte en contenido formativo si nos toca (nous touche), ser-tocado, concierne en nuestro Ser, a cómo nos cautiva, transforma, y es desde la acción mediadora como se encamina el contenido hacia un sentido, hacia una dirección. En Heidegger el camino que interroga por el sentido del Ser está justamente ante nosotros. Y precisamente, porque está próximo, lo encontramos con dificultad.

Hasta ahora, hay una relación entre problema-objetivo-contenido- es entonces necesario emprender un camino -método-, que permita una vía hacia el eidos o esencia de la paideia. Ir tras la esencia de la educación implica tomar el camino de la hermenéutica, pues en la teoría de la formación categorial interesa acceder al sentido que tienen las acciones pedagógicas, pues no hay modo de pensar en el hecho educativo sin referencia a su para qué. Al fin y al cabo, siempre que se educa, se educa a alguien, para algo. Este para algo es consustancial a la acción formadora y este para algo es aquello que se revela en el para qué -relacionado con los objetivos- o con los fines de la educación.

Algunos autores coinciden en que el método tiene relación con los objetivos y los contenidos. Para Klafki (1990: 90) los métodos sólo se pueden discutir y decidir cuando se han tomado decisiones sobre objetivos y contenidos; Blankertz (citado por Klafki, 1990: 96) utiliza el concepto de método en un doble sentido. Por una parte, en relación con las formas de organización de la enseñanza y el aprendizaje en la clase y, por otra parte, emplea este concepto de tal manera que con él se designa un aspecto de la dimensión de las decisiones sobre objetivos.

Al camino del sentido se va por la ruta de la hermenéutica, que utiliza métodos comprensivos que aluden a lo que señala Copei (citado por Speck y Wehle, 1981: 294) "Enseñar no significa comunicar algo, 
sino preparar el momento fecundo, despertar una viva disposición, que en lucha con el objeto aspira a asimilarse el contenido de sentido". Un método comprensivo debe hacer posible que el estudiante se enfrente con un contenido que esté orientado a un fin (telos), a un para algo; en consecuencia, para Klafki (1990), los métodos de enseñanza en la teoría de la formación categorial no pueden ser entendidos como meros instrumentos, sino como formas en las cuales se relacionan entre sí, por una parte, procesos de enseñanza y por otra, procesos de aprendizaje.

Además, la 'metáfora del salto' lleva a que en el proceso docente-educativo se ponga al descubierto un camino que también conduzca al Ser, y para ello el método sólo puede entenderse como 'poner', 'disponer', ponerse en camino.

\section{4. ¿ESTAMOS EN CAMINO?}

Los leñadores y guardabosques conocen los caminos.

Ellos saben lo que significa encontrarse en un camino que se pierde en el bosque...

Heidegger (2003: 10)

Al final de este viaje por el bosque, se encuentra que para emprender el camino de la didáctica habrá que partir de una teoría de la formación; esto hará que la didáctica no quede reducida a lo estrictamente instrumental, pues sería una versión reducida poner la didáctica a un sólo hacer, a un 'cómo', a un saber normativo dedicado al dominio de técnicas, metódicas y fórmulas mecánicas que operacionalizan la enseñanza y el aprendizaje de un saber.

El contenido es importante en la medida en que cobra sentido, en tanto se articule desde una perspectiva pedagógico-formativa y un camino es la hermenéutica, porque favorece la interpretación, la comprensión y otorga algo significativo para el ser-que-se-forma. En palabras de Klafki, es significativo 'cuando un contenido (Inhat) tiene contenido formativo (Gehalt)'.

Tender hacia una educación de las 'fuerzas corporales' implicaría de-construir las formas de entender el movimiento humano y la corporalidad en la formación, anclados en un discurso racionalista y cartesiano, donde el movimiento humano pase de ser visto como una clase de movimiento que se explica desde la psicofisiología de un modo exclusivamente mecánico, por un movimiento vivido ${ }^{7}$ en la perspectiva cuerpo-sujeto, donde el movimiento corporalizado permita modos-de-llegar-a-ser-en-el-mundo.

¿Para qué le sirve a un individuo aprender el movimiento mecánico, ¿qué lugar ocupa el contenido formativo?, ¿qué tipo de sujetos crea una educación que privilegia el cuerpo-objeto y el movimiento objetivo? Quizás, se despoja, en gran medida, de subjetividad y de su carácter formativo como aquel que pone en claro los presupuestos antropológicos de la formación, fija el camino que tiene que recorrer el hombre en el proceso de hacer-se, que como ser humano necesitado de educación tiene la capacidad de formar-se.

Si la Educación Física sigue privilegiando los contenidos conducentes al cuerpo-objeto (Körper) que se mueve para mejorar la capacidad física, adquirir destrezas motoras, desarrollar capacidades y

\footnotetext{
${ }^{7}$ Según Straus (1966: 69-70) movimiento vivido es "cambio que tiene lugar en algo que es ello mismo cambiable, esto es, un llegar-a-ser...El problema del movimiento vivido está incluido en el fenómeno básico del cambio, en el llegar-aser mismo. Querer moverse sólo le es posible a un sujeto que al estar él mismo incluido en el llegar-a-ser, llega a ser diferente".
} 
habilidades motrices, desarrollar competencias prácticas desde un gran repertorio de actividades como formas jugadas, danzas, gimnasia, deportes, entre otras; la pregunta seguiría en camino, ¿quedan en estos contenidos el sentido del movimiento del ser-que-se-mueve y de la corporalidad a la luz de las teorías de la formación?

\section{BIBLIOGRAFÍA}

FoucAult, Michel. La Hermenéutica del sujeto. Buenos Aires: Fondo de Cultura Económica, 2002.

FULLAT, Octavi. Antropología filosófica de la educación. Barcelona: Ariel, 1997. Filosofías de la educación. PAIDEIA. Barcelona: Ceac, S.A, 2000.

GADAMER, Hans-Georg. El giro hermenéutico. Madrid: Cátedra, 2001. . La educación es educarse. Barcelona: Paidós, 2000.

GALlo, Luz Elena. "Una mirada de la corporalidad en la formación". En: Un siglo de vida en Medellín. Fundación VISTAZ, 2004. Formato digital, ISBN 958-97444-4-3.

GeHlen, Arnold. Antropología filosófica. Del encuentro y descubrimiento del hombre por si mismo. Barcelona: Paidós, 1993 [1986].

GRUPE, Ommo Estudios sobre una teoría pedagógica de la educación física. Madrid: Instituto Nacional de educación física, 1976.

HeIDEgGer, Martín. Caminos de Bosque. Madrid: Alianza, 2003 [1984] ¿Qué significa pensar? Buenos Aires: Nova, 1978. p 9-23.

KLAFKI, Wolfgang. "Sobre la relación entre didáctica y metódica". Traducido del alemán por DE MESA, Alicia y PANTOJA, Alvaro. En: Revista Educación y Pedagogía, Vol 2 № 5, octubre de 1990-enero de 1991, Universidad de Antioquia, Facultad de Educación. p 85-108.

“¿Pueden contribuir las ciencias de la educación a la fundamentación de objetivos pedagógicos?”. En: revista Educación Vol 45 editada por el instituto de colaboración científica, Tübingen, Alemania, 1992 p. 39-51.

- "La importancia de las teorías clásicas de la educación para una concepción de la educación general hoy". En: revista Educación Vol 36 editada por el instituto de colaboración científica, Tübingen, Alemania, 1987 p. 40-65.

MEINBERG, Eckhard. "El valor posicional de la antropología en la formación de la teoría sobre la educación física y el deporte escolar". En: Revista Educación Vol 47 editada por el instituto de colaboración científica, Tübingen, Alemania, 1993 p. 62-83.

Merleau-Ponty, Maurice. Fenomenología de la percepción. Barcelona: Península, 1975 [1945].

NoHL, Herman. Antropología pedagógica. México: Fondo de cultura economía, 1954 [1938].

PLATÓN. Apología de Sócrates. En: Diálogos. Madrid: Gredos, 1997.

Rodriguez Rojo, Martín. Hacia una didáctica crítica. Madrid: La Muralla, 1997. 
Roth, Henrich. "El buen arte de la preparación de la clase". En: revista Educación Vol 1 editada por el instituto de colaboración científica, Tübingen, Alemania, 1970 p. 26-36.

RUNGE, Andrés Klaus. Aspectos fundamentales de la pedagogía crítico-constructiva y de la didáctica teóricoformativa de Wolfgang Klafki. (Documento en preparación), 2004.

SPECK, Josef; WEHLE, Gerhard. Conceptos fundamentales de pedagogía. Barcelona: Herder, 1981.

Straus, Edwin W. Psicología fenomenológica. Buenos Aires: Paidos, 1996.

WULF, Christoph. Antropología de la educación. Barcelona: Idea Books, S.A, 2004.

WULF, Christoph. "Antropología histórica y ciencia de la educación". En: revista Educación Vol 54 editada por el instituto de colaboración científica, Tübingen, Alemania, 1996 p. 84-92. 


\title{
Contactar
}

Revista lberoamericana de Educación

\author{
Principal OEI
}

